\title{
STUDY ON PHOTOELECTRIC AND DYNAMICAL CONTROL SYSTEM FOR FRUIT SIZING
}

\author{
Dongxing Zhang and Jing Li \\ College of Engineering, China Agricultural University P.O. Box 46, Beijing 100083
}

\begin{abstract}
A fruit sizing system based on photoelectric sensor is developed. The system uses AT89C51 microcomputer as its core, and a signal gathering circuit, a processing circuit, an output circuit and a display/keyboard unit are also provided. When it works, the system receives digital signals produced by fruits that shadow the light from a photoelectric sensor. After digital signals processed by the system software, every fruit's sizing level is deduced. Then, the system will output switch signals to open the sorting switches according to fruits' size, and fruits are dynamically measured and sorted in real time. Experiment results indicated that the system works well and can be easily controlled; meanwhile it is multipurpose by setting sizing levels to sort different kinds of fruits.
\end{abstract}

Key words: microcomputer, photoelectric sensor, fruit sorting

\section{INTRODUCTION}

Fruit production are developing very fast In China in recent years, and the increased consumers' awareness of fruit quality has made fruit production and marketing very competitive. Although growers always try to select optimal fruit varieties, and use the correct practices, they do not have absolute control over their products and therefore, unlike other industries, the "production line" of fruit will produce very non-uniform products. There has been an increasing demand for tools to sort and grade those products by various criteria. Such tools will enable the growers to grade their products 
and to predict the shelf life and direct the various grades to different markets according to their transportation schedule or predicted revenues.

Meanwhile, huge quantities of fruits are used by food industry. Food processing plants manufacture more than one product and use fruits of different quality for different products. For example, peach-processing plant may use firm peaches for its canning production, and soft and overripe ones for its juice and paste line. Sorting and grading the fruits will enable the processors to direct their optimal use and save a great deal of money.

When fruits are harvested, they must be cleaned, sorted, sized, graded, and packed according to its destination. Those operations are likely to be done as close as possible to the field. Quite often preparation of the products for marketing includes additional steps such as wax coating, disinfection, or drying. All these operations are performed to increase the value and marketability of the products, and in many cases some of these operations are required in order to conform to marketing regulations and standards.

Fruits are usually sorted according to their "Quality". Two main quality categories are widely used: external and internal. The "external" attributes include size, weight, shape, and color; the "internal" attributes include sugar, water content, acidity, firmness and tissue structure, bruise, tissue breakdown, internal color, etc.

Though external attributes are generally used to sort fruits, but in most cases the internal and external quality attributes are closely related. For example, an overripe fruit may change in color, sugar content, firmness and may show rot spots on the peel. A small fruit is normally not to be mature enough. A bruised fruit may show external color changes.

\section{INTRODUCTION}

\subsection{Sizers}

Belts: It uses diverging belts on which fruits travel until the gap between the belts is wide enough for the fruits to pass through. Another version is the belt-and-bar sizer. On this one, the belt conveyor is inclined at a side angle so that fruits are forced to roll against a fixed bar which creases a gap, increasing in size. When the gap is large enough the fruit rolls under the bar and into a bin. Fruit may be sized by any of its dimension. The result may be poor sizing accuracy.

Bars: Parallel bars are used to rotate the fruits about their axis and cause orientation of the fruits. This method is good with fruits such as lemons or oranges. The rotation of the fruits is achieved by rotating the parallel bars. In 
some cases the bars are fixed in their position and the fruits are pushed forward by incoming fruits and thus progress along the sizer. Alternatively, the bars are linked into an infinite chain that is driven as a conveyor.

Cylinders: There are 3 or 4 cylinders on the sizer, different diameter holes are allocated on each cylinder. Fruits are driven to pass the cylinders. If the size of a fruit is smaller than the holes on a cylinder, this fruit will pass the hole falling into to a tray. Otherwise, it will move to next cylinder, and so on.

\subsection{Weight sortor}

Apples, pears, tomatoes, and other sensitive fruits are sometimes weighted rather than sized. Fruits are placed in individual trays on a trayconveyor, which slide over a weighing sensor monitored by a computer, which controls the discharge of the fruits into weight groups. Mechanical system is sometimes used instead of electrical system, but less accurate.

\subsection{Mchine vision}

Machine vision, also named computerized vision or artificial vision, is a sensing method in replacing the human visual inspection. Cameras and sensors are used for fruits features extraction and classification, algorithms for images processing and analysis. Although machine vision methods detect external visible quality attributes, some of the measurements may give indications of the overall quality of the products.

Machine vision system will have a bright future as fruit sorters, since hardware costs are declining rapidly, and the advantages of a combined inspection result of other quality attributes such as size, shape and col

\section{PHOTOELECTRIC AND DYNAMICAL CONTROL SYSTEM FOR FRUIT SIZING}

\subsection{System Hardware}

Signal Gathering System: 2 infrared transducers are oppositely positioned on the fruit sorting unit, one to sent light signals, well the other receives signals.12 36 voltage input, and high \& low switch signals output. 
Signal processing System: Signal and data processing system is the core for the sizer. It will control all the activity of the machine. Its performance will affect working speed, accurate, reliability and efficiency. Ether a PC or a single chip can be used, but PC will be cost and complicate to operate. Single chip AT89C51 is selected due to its advantages: low cost, high reliability, simple to program \& erase, easy to expand, small in size etc.

Signal Output System: LED and LCD are used to display the output results, and executive orders will be sent to electromagnetic valves.

\subsection{System Software Modules}
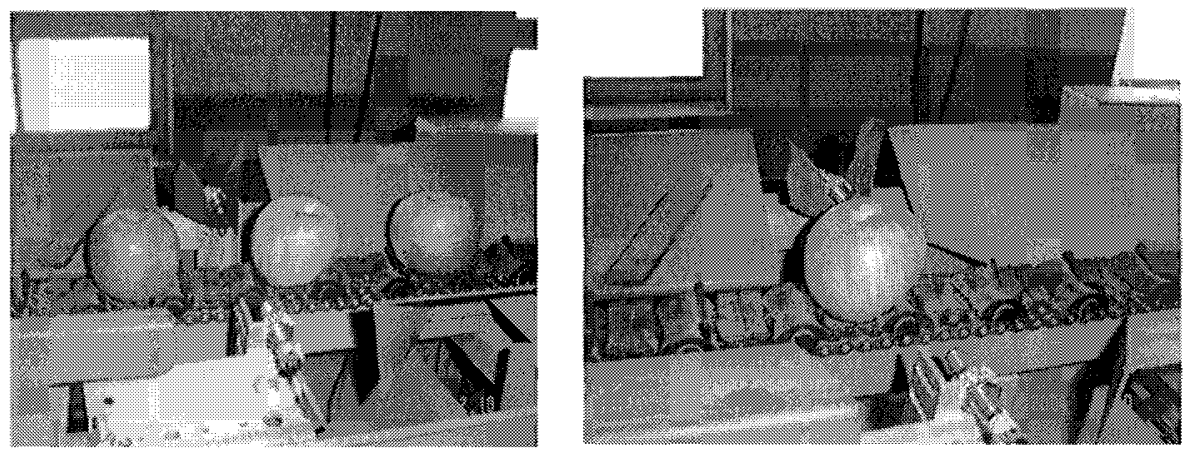

Figure 1 Infrared sensors

Working principle of the sizer: When a fruit passes through the 2 sensors (figure 1), it will shade the light signal sent by one of the 2 sensors, so the other sensor could not get the light signal, makes the sensors output voltage changes, resulting single chip outside interrupt, entering into data processing system. When interrupt happens, timing program of the single chip is initiated, which will inspect the system intermittently. Meanwhile, the data processing system will start to grade the fruit into a "group" according to fruit "size", sending an executive order to the relative electromagnetic valve to open for discharging this fruit to that group.

Signal Gathering program Module: As descript above, shading light will make the sensors output voltage changes. In reverse, when fruit left the sensors, it will also cause voltage changes, and the timer will stop working. According to the time it takes for shading light, and the belt velocity, fruit' "size" could be calculated:

$$
S=V \times T
$$




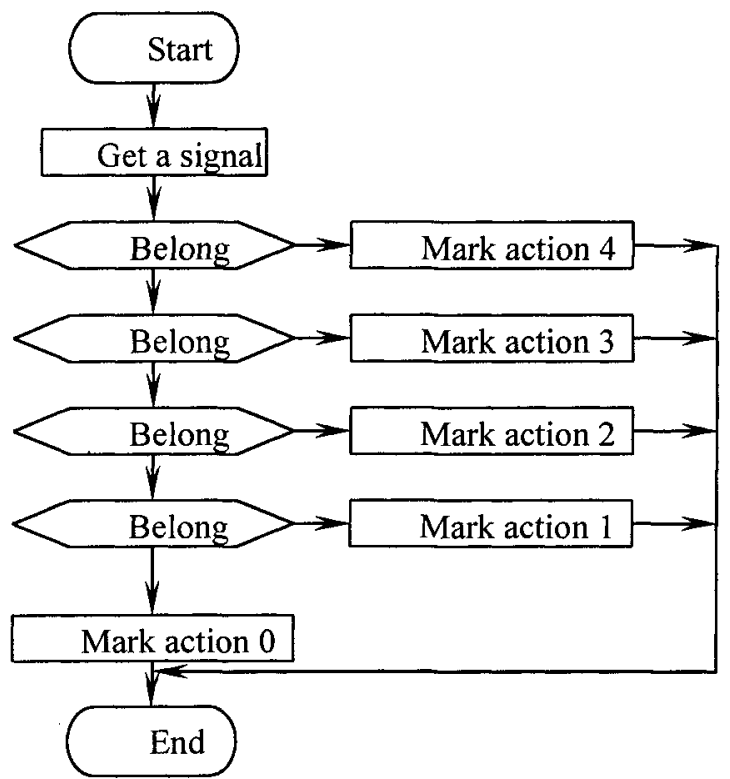

Flow chart 1 Data Processing chart

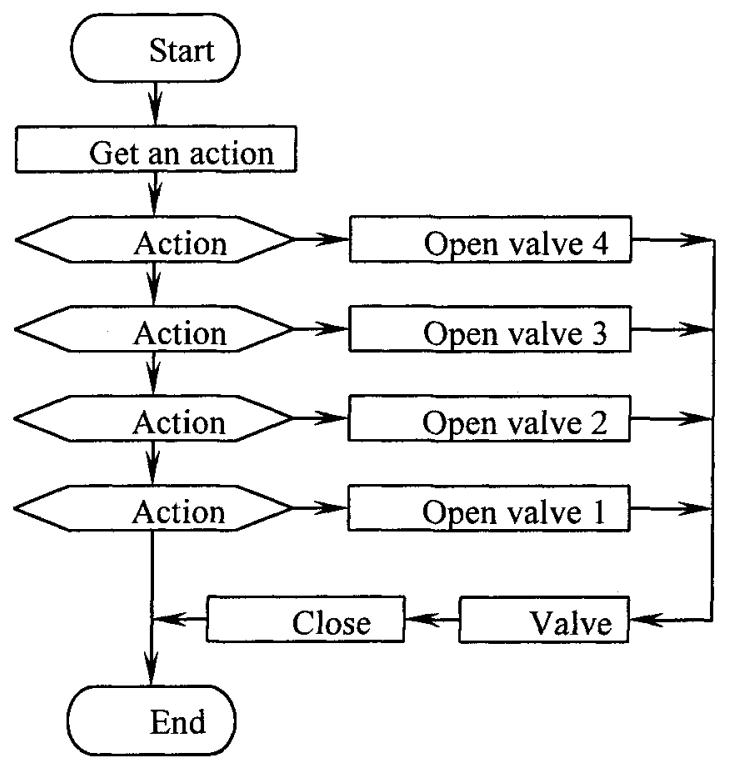

Flow chart 2 execute control chart 
Signal Processing program Module: Before starting the system, the number of grades for fruit sorting must be preset first. It is completely artificial, you can set as many grades as you like, but 4 grades are popular in practice.

When processing program runs, it will compare a gathered signal with the 4 presetting grades one by one. If this signal belongs to grade 4 , an action 4 will be marked. If the signal is smaller than grade 4 , then it will be compared with grade 3 , and so on, till the equivalent grade for this signal is found (flow chart 1). After calculation and comparison done by the processing program, all the gathering signals can be marked with action numbers, so all fruits can sorted into relative grades.

Executive control program Module: When the system works, the program will send discharge orders to the relative electromagnetic valves according to the different action marks (flow chart 2).

Man-Machine communication function: This makes it possible for one to preset the number of grades, and it will also display the sorting results, so one can directly look at the operation status.

\section{ORIENTING SYSTEM}

In order to get higher sorting accuracy, an orienting unit is developed. When it works, the orienting wheel is turning vertically while the cup is turning horizontally. Once the stem or calyx area touched the orienting wheel, the fruit was in a strait up \& down position. Then the sensors could measure the maximal diameter of a fruit.

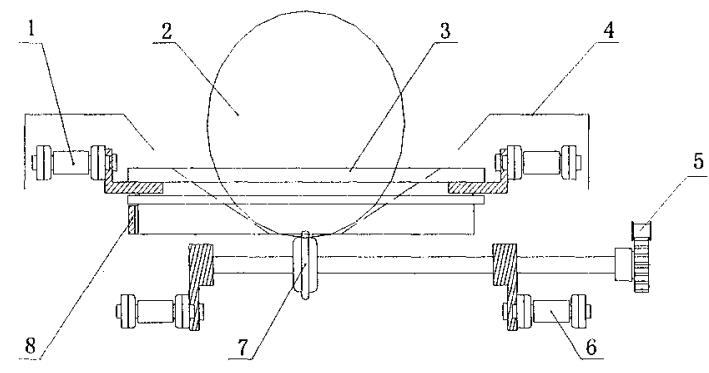

Fig.2 The Apple Orientation Unit 


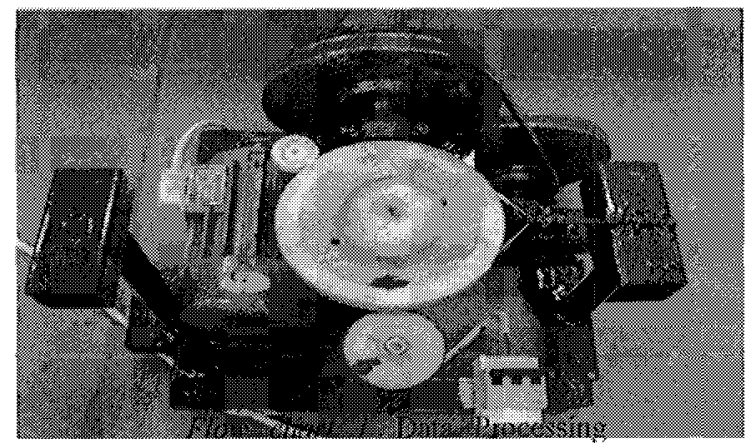

\section{CONCLUSION}

Infrared sensors and single chips are used for the system instead of weighing sensors and $\mathrm{PC}$, that reducing the system cost and making the system simplified significantly, experiment results indicate that there is no compromising of fruit sorting quality.

\section{ACKNOWLEDGEMENTS}

The authors would like to thank Mr. Wang Xinting, Mr. Zhang Hailiang, Mr. Xie Feng and Miss Zhou Qinghong for their significant contribution to the machine system

\section{REFERENCES}

[1] Jose Campins, James A. Apple Stem and Calyx Indentification for Automatic Sorting. ASAE Paper, No.373079, 1997

[2] Dr.Boaz Zion. Principles of Sorting, Sizing and Grading. Postharvest Technology, 1994

[3] Wang Xinting, Zhang Dongxing. Study on Single Chip Control and Measurement System for Fruit Sorter, Journal of China Agricultural University, No.4 Vol.8, 2003 